Reprod. Nutr. Dévelop., 1985, 25 (4 B), 763-775.

\title{
Contrôle nerveux de la motricité du réticulo-rumen
}

\author{
J.-P. ROUSSEAU, M. FALEMPIN
}

Laboratoire de Neurophysiologie Végétative, LA 308, CNRS, Bat. SN4, Université de Lille l, 59655 Villeneuve d'Ascq Cedex.

Summary. Nervous control of reticulo-ruminal motility in ruminants.

This paper reviews the nervous mechanisms involved in the control of motility of the forestomach compartments in ruminants. The first part of the review reports the efferent vagal discharge which consists of several distinct and independent types of unitary activity and passes from the gastric centres to the reticulo-rumen. The patterns of each of these activities are temporally related to the contractions of special parts of the forestomach and occur in a sequence which could produce the coordinated series of movements found in the reticulum and rumen. The orderly sequence of motor events that constitutes the gastric cycle is due to this coordination of efferent vagal outputs arising in the gastric centres. The focal point of the second part of the paper is sensory feedback from the complex stomach to the centres. Four types of receptor have been identified according to their location and stimulus; these are tension receptors and epithelial receptors in the reticulo-rumen and tension receptors and mucosal receptors in the abomasum. Mechanical or chemical stimulation of these distinct receptor types leads to either facilitation or inhibition of reticulo-ruminal motility. The third part of the paper deals with the organization of the medullary gastric centres. The gastric vagal motoneurons are controlled by interneurons organized in two functionnaly distinct networks. The " rate " network for which the periodicity of its activity depends on the cumulative integrated afferent inputs from central and peripheral sources, determines the rhythm of gastric cycles. It is postulated to drive the " amplitude and form " network which adjusts vagal output to instantaneous gastric afferents, enabling the amplitude of gastric contractions to be adapted to peripheral stimulations. The role of the sensory feedback from the complex stomach in the control of the "frequency " network is discussed, taking into account new experiments on vagal deafferentation and the concept of an oscillating generator that would be more or less permanently inhibited by vagal afferents is reviewed. The respective roles of local regulation mechanisms, mediated in the intramural plexus, and of central mechanisms, in the control of forestomach motility, are briefly discussed.

Le but de ce rapport est de faire le point sur les mécanismes nerveux mis en œuvre dans la régulation de la motricité des réservoirs gastriques chez les Ruminants. On sait que le cycle moteur des préestomacs débute par la contraction biphasique du réseau suivie par la contraction des piliers et du sac dorsal du rumen et enfin par celle de son sac ventral. Cette séquence de contractions, encore appelée cycle primaire ou séquence $A$, dure de 15 à $25 \mathrm{~s}$ et se répète selon une organisation identique, au rythme d'environ un cycle par 
minute. Son rôle est d'assurer le mélange des contenus, de faciliter leur dégradation microbienne, de permettre le transit des particules les plus fines vers les compartiments postérieurs. Le rythme des cycles primaires varie avec la nature du régime, la prise alimentaire, le comportement mérycique, la réplétion gastrique. Un cycle primaire peut être suivi d'un cycle secondaire ou séquence B de contractions, au cours duquel seul le rumen est impliqué. Cette séquence débute par la contraction du sac caudal ventral ; une contraction du sac dorsal lui fait suite, qui déplace les gaz vers l'avant jusqu'au cardia et permet l'éructation. La fréquence des cycles secondaires augmente avec l'état de distension du rumen. Les mouvements gastriques dépendent de l'intégrité des nerfs vagues. La section des troncs œesophagiens dorsal et ventral dans le thorax abolit ces contractions primaires et secondaires. Celles-ci sont qualifiees d'extrinsèques par opposition aux contractions intrinsèques qui persistent après bivagotomie et qui reflètent l'état d'excitabilité des plexus myentériques. La disparition des contractions extrinsèques et l'absence d'une organisation séquentielle des contractions intrinsèques chez les animaux bivagotomisés suggèrent un strict contrôle du système nerveux central sur la périphérie. En retour, les modifications de l'amplitude et de la fréquence des contractions, induites par des stimulations du réticulo-rumen démontrent l'existence d'afférences spécifiques capables de moduler l'activité nerveuse centrale. Nous nous proposons donc d'étudier successivement : (i) l'activité efférente des fibres vagales qui innervent le réticulo-rumen, (ii) l'activité des récepteurs gastriques et la nature de leur stimulus, (iii) l'organisation des centres gastriques et leur dépendance vis-à-vis de l'information d'origine périphérique. Dans une dernière partie, nous ferons état des approches récentes concernant les réflexes locaux qui se consomment au sein des plexus intrapariétaux et les relations possibles entre les deux niveaux de régulation, central et périphérique.

\section{Activités des efférences vagales associées aux contractions du réticulo-rumen}

Dussardier $(1958,1960)$ est le premier a avoir recueilli l'activité des neurones vagaux innervant les préestomacs chez le mouton. A la suite d'une suture entre l'extrémité centrale d'un nerf vague sectionné dans son trajet thoracique et l'extrémité périphérique du nerf phrénique ipsilatéral, les axones des neurones vagaux qui innervaient à l'origine les derniers centimètres de l'œsophage et les viscères abdominaux sont capables de réinnerver l'hémidiaphragme correspondant. Neuf à douze mois plus tard, des décharges d'unités motrices peuvent être obtenues pendant des sessions d'enregistrement chez l'animal éveillé, en implantant une électrode bipolaire dans l'hémidiaphragme réinnervé, qui avait été préalablement transplanté sous anesthésie générale en position souscutanée. Certaines d'entr'elles traduisent l'activité de neurones vagaux qui innervaient à l'origine le réticulo-rumen. Chez le mouton anesthésié, l'activité nerveuse est recueillie directement soit en implantant des micro-électrodes dans le bulbe, dans le noyau dorsal du vague, au voisinage des corps cellulaires des neurones gastriques (Beghelli, Borgatti et Parmeggiani, 1963 ; Howard, 1968), 
soit en plaçant des électrodes bipolaires sur l'extrémité centrale des fibres isolées par la dissection fine du nerf vague cervical (Leek, 1963 ; lggo et Leek, 1967). Une unité recueillie par une de ces trois méthodes doit satisfaire à plusieurs critères pour être considérée comme gastrique. En d'autres termes, sa décharge traduit l'activité d'un neurone qui innervait le réticulo-rumen : (i) si elle apparaît, ou est modifiée dans le cas d'unités toniques, au même moment de chaque cycle primaire des réservoirs gastriques, (ii) si, pour les unités phasiques, elle ne se produit pas pendant la phase des cycles au cours de laquelle aucun évènement moteur n'est observé, (iii) si elle varie dans ses caractéristiques avec les changements spontanés ou induits de l'amplitude et de la fréquence des contractions. Le résultat essentiel est que les neurones dont l'activité est associée aux contractions du réticulo-rumen sont activés à des temps différents par rapport au début du cycle primaire. Selon les caractéristiques et surtout le moment de leur décharge au cours du cycle, sept catégories de neurones sont ainsi décrites par lggo et Leek (1967). Sans entrer dans le détail de cette classification, on peut souligner que les unités précoces qui apparaissent avant et/ou pendant la contraction biphasique du réseau traduisent la décharge de neurones qui innervaient le réseau et/ou des structures qui lui sont associées, telles que le pli réticulo-ruminal ou l'orifice réticulo-omasal. Les unités qui sont activées pendant et après la seconde phase de la contraction réticulaire, avec un retard plus ou moins accentué selon les unités, correspondent aux neurones innervant le rumen antérieur, dorsal ou ventral ainsi que les piliers. Les contractions successives qui constituent chaque cycle primaire résultent donc de la mise en jeu séquentielle et ordonnée de neurones vagaux, commandant les différentes structures composant le réticulo-rumen. II a été également montré que les mêmes neurones dont l'activité pendant les cycles primaires est associée aux contractions du rumen dorsal et vraisemblablement du pilier antérieur, sont aussi mis en jeu pendant les cycles secondaires. Les caractéristiques de leurs décharges présentent alors une plus grande variabilité qu'au cours des cycles primaires. Elles traduisent cependant une rigoureuse organisation du contrôle des structures du rumen mises en jeu pour permettre l'éructation (Rousseau, 1984).

Ces résultats montrent que l'organisation séquentielle des contractions gastriques chez le mouton est largement dépendante d'une programmation centrale bulbaire, mais ne permettent pas d'écarter totalement l'existence d'une organisation intrinsèque de la motricité, au sein des plexus myentériques. Une telle organisation qui permettrait la mise en jeu, par l'activité vagale efférente, de voies plurisynaptiques plus courtes pour le réseau et les structures anatomiquement voisines, de plus en plus longues pour le rumen dorsal, ventral et caudal, $n$ 'est cependant pas démontrée. Cette hypothèse, proposée par Morrison et Habel (1964), a été essentiellement fondée sur l'observation, insuffisante, que les fibres préganglionnaires vagales attaquent les neurones postganglionnaires des plexus dans une seule région dite " centrale ", localisée dans la partie antérieure des réservoirs gastriques. Elle n'est pas confirmée par les expériences de vagotomie : les contractions phasiques qui apparaissent à partir de deux semaines après la bivagotomie restent en effet peu ou pas coordonnées (Ruckebusch, Tsiamitas et Bueno, 1972 ; Gregory, 1982). 
Un dernier point concernant l'interprétation des résultats mérite d'être soulevé. Les neurones vagaux dont l'activité est associée aux contractions du réticulo-rumen sont généralement considérés comme moteurs ; on déduit en effet de la relation temporelle activité-contraction, qu'ils font normalement synapse avec des neurones postganglionnaires cholinergiques excitateurs. Or, le relâchement de structures sphinctériennes telles que l'orifice réticulo-omasal dépendrait plutôt de la stimulation, par l'activité vagale efférente, de neurones postganglionnaires inhibiteurs non-cholinergiques, non-adrénergiques, dont le neuromédiateur pourraît être le vasoactive intestinal peptide (VIP) (Newhook et Titchen, 1972 ; Reid et Titchen, 1984). Des unités peuvent ainsi traduire l'activité de fibres vagales faisant normalement synapse avec de tels neurones postganglionnaires inhibiteurs. Les unités de type III de lggo et Leek (1967) pourraient être interprétées dans ce sens, car l'augmentation de leur décharge se produit pendant la contraction réticulaire, à un moment où l'orifice réticulo-omasal est ouvert pour permettre le transit des particules. II importe donc à l'avenir d'identifier avec plus de certitude et de précision les structures gastriques qu'innervent les neurones dont l'activité est recueillie en association avec les mouvements gastriques. La contraction et le déplacement des piliers, le diamètre des orifices entre les compartiments sont des paramètres à prendre en compte, en plus des contractions du réseau ou du rumen classiquement enregistrées dans ce type d'étude.

\section{La sensibilité gastrique}

Deux approches expérimentales complémentaires permettent d'étudier la sensibilité des préestomacs :

i) la mise en évidence de réflexes gastriques: différents stimulus appliqués à l'estomac sont à l'origine de réflexes longs vago-vagaux qui modifient le rythme et l'amplitude des contractions :

ii) l'enregistrement de l'activité unitaire des afférences vagales d'origine gastrique : il est obtenu, chez le mouton anesthésié à partir de l'extrémité périphérique de fibres vagales isolées par dissection fine et sectionnées au cou (Iggo, 1955; Leek, 1969, 1971), ou grâce à des microélectrodes implantées dans le ganglion plexiforme au voisinage des corps cellulaires des neurones sensitifs (Falempin, Mei et Rousseau, 1978). Chez le mouton éveillé, on peut recueillir l'activité d'un récepteur en faisant réinnerver un muscle du cou par les axones sensitifs vagaux, à la suite d'une anastomose entre la racine centrale du ganglion plexiforme et l'extrémité périphérique du nerf spinal-accessoire, nerf moteur de ce muscle. Nous avons démontré que l'activité d'une unité motrice réinnervée traduit alors celle d'un récepteur du territoire vagal (Rousseau et Falempin, 1979, 1984 ; Falempin et Rousseau, 1983). Cette technique permet l'étude des récepteurs digestifs dans les conditions physiologiques, alors que la motricité gastrique est perturbée, voire abolie dans les conditions expérimentales aiguës qu'imposent les deux méthodes précédentes. Elle aboutit cependant à un tri sévère des types d'unités étudiées, puisque seuls les axones sensitifs cholinergiques ou potentiellement cholinergiques peuvent réinnerver. 
La plupart des réflexes gastriques s'expliquent par l'existence de 4 types de récepteurs identifiés selon la nature de leur stimulus et leur localisation (revues dans Leek, 1971 ; Leek et Harding, 1975).

1. - Les récepteurs de tension du réticulo-rumen sont des mécanorécepteurs à adaptation lente. Ils sont surtout localisés dans la partie la plus antérieure des préestomacs avec une plus grande densité dans les parois du réseau et du sac cranial du rumen. II est remarquable de noter que peu de récepteurs de ce type sont trouvés dans les régions postérieures du rumen où naissent les contractions secondaires éructatives. Ces récepteurs, à bas seuil, sont stimulés par les augmentations de la tension pariétale, que celles-ci soient provoquées par l'étirement des parois lors d'une distension passive ou induites activement par les contractions. Ils présentent des seuils d'excitation différents : pour un tonus musculaire faible, certains ne montrent pas de décharge de base entre les contractions extrinsèques, alors que la plupart pulsent de façon intermittente, en bouffées rythmées vraisemblablement sur les contractions localisées intrinsèques du viscère. L'augmentation du tonus du viscère fait apparaître une décharge de base irrégulière chez les premiers; celle des secondes devient alors continue. Les récepteurs de tension sont vraisemblablement localisés dans la couche musculaire, " en série " avec les éléments musculaires selon le concept classiquement admis, mais aucune localisation histologique ne permet de confirmer cette hypothèse. Ils sont en relation avec des fibres dont la vitesse de conduction est de l'ordre de $12 \mathrm{~m} . ~ s e c^{-1}$ (Leek, 1969). Leur stimulation entraîne une augmentation de la motricité gastrique; elle est à l'origine des réflexes excitateurs déclenchés par la distension du réseau ou par l'étirement du pli réticulo-ruminal, tant chez l'animal éveillé (Ash et Kay, 1959 ; Dussardier, 1960) que dans les conditions expérimentales aiguës (Titchen, 1960 ; Dussardier, 1960 ; Iggo et Leek, 1967). Ces récepteurs peuvent également renseigner sur le degré de remplissage des réservoirs puisque la fréquence de leur décharge de repos est fonction de la tension pariétale.

2. - Les récepteurs de tension de la caillette se comportent comme ceux du réticulo-rumen. Ce sont aussi des mécanorécepteurs à adaptation lente, à bas seuil, en relation avec des fibres de conduction plus lente. Leur stimulation peut être à l'origine de l'inhibition des contractions du réticulo-rumen (Titchen, 1958 ; Ruckebusch et Kay, 1971) et de celle de l'activité vagale efférente gastrique (Harding et Leek, 1972b), observées lors de la distension abomasale. Le remplissage de la caillette, en réduisant la motricité des préestomacs par une telle rétroaction négative, provoquerait la limitation du transit des contenus vers les compartiments postérieurs dont la surcharge serait ainsi évitée.

3. - Les récepteurs épithéliaux du réticulo-rumen sont sensibles à la fois a des stimulations mécaniques et chimiques. Leur distribution dans le réticulorumen est plus étendue que celle des récepteurs de tension. Dans le réseau, leur champ récepteur est limité à environ cinq parois d'une même alvéole, soit à la douzaine de papilles coniques qui les surmontent (Harding et Leek, 1982a). L'histologie montre dans chacune de ces papilles la présence d'une fibre extrêmement fine qui se divise en plusieurs terminaisons libres (Leek, 1972). La stimulation de l'épithélium en le brossant localement avec les poils d'un pinceau, la distension exces- 
sive du réseau - en fait le frottement de l'épithélium par la paroi du ballon en contact étroit avec lui par suite de l'importance de la distension - entraînent dans la fibre afférente des décharges qui traduisent une adaptation rapide des terminaisons aux stimulus mécaniques. Ces récepteurs polymodaux sont également sensibles aux solutions acides, alcalines, hypo- et hyperosmotiques. En ce qui concerne les solutions acides et alcalines, le stimulus est leur acidité ou leur alcalinité titrable plutôt que leur $\mathrm{pH}$. Les concentrations seuils d'acides gras volatils (AGV) sont en moyenne de 40 à 50 mmoles. $1^{-1}$, mais varient d'un récepteur à l'autre dans une large gamme, de 20 à 100 mmoles . ${ }^{-1} \mathrm{~d}^{\prime} \mathrm{AGV}$ (Harding et Leek, 1972a ; Crichlow et Leek, 1980 ; Crichlow et Chaplin, 1984). L'efficacité des solutions acides est d'autant plus grande que le poids moléculaire de l'acide est plus faible (Harding et Leek, 1972a). Pour d'autres auteurs, les récepteurs étudiés sont au contraire plus sensibles à l'acide butyrique qu'aux acides acétique et propionique (Crichlow et Chaplin, 1984). La signification physiologique d'un tel équipement sensoriel apparaît évidente lorsqu'on aura rappelé que l'acidification du contenu ruminal entraîne l'inhibition de la motricité gastrique (Ash et Kay, 1959). Les perfusions par des solutions d'AGV dont les concentrations, selon les acides, sont de 20 a 30 mmoles.$I^{-1}$ inhibent seulement les contractions primaires du rumen. II faut des concentrations nettement supérieures, de 35 à 100 mmoles $.1^{-1}$, pour que soient inhibées les contractions secondaires du rumen et les contractions du réseau chez le mouton intact, les contractions locales intrinsèques chez le mouton bivagotomisé (Gregory, 1984b). Par ailleurs, l'acide butyrique semble le plus efficace (Leek, Ryan et Upton, 1978 ; Gregory, 1984b). Ces récepteurs sont donc très sélectifs, à la fois par le large spectre de leurs seuils de sensibilité et par la spécificité de cette sensibilité : l'augmentation globale de la concentration des AGV dans le réticulo-rumen aboutit à un recrutement de plus en plus large des récepteurs; le résultat en est l'accroissement progressif des entrées afférentes chémosensibles dans les centres gastriques et l'inhibition successive des contractions des différentes poches (réseau ou rumen) ou de différents types (primaires ou secondaires). Ce processus est obtenu plus rapidement si l'acide butyrique domine dans le jus de rumen. Enfin, les récepteurs à bas seuil sont vraisemblablement stimulés en permanence par les concentrations d'AGV habituellement observées dans le réticulo-rumen et déterminent par voie réflexe un effet inhibiteur tonique de base sur sa motricité.

4. - Les récepteurs muqueux de la caillette répondent comme les récepteurs épithéliaux du réticulo-rumen. Leur seuil d'excitation aux stimulus mécaniques est bas, ce qui explique que les mouvements de va-et-vient d'un ballon dans la caillette stimulent plutôt ces récepteurs que les récepteurs de tension. La réponse réflexe à ce type de stimulation est effectivement une augmentation de la motricité du réticulo-rumen et non son inhibition (Harding et Leek, 1973). Ces récepteurs sont aussi sensibles aux acides chlorhydrique et acétique. Dans les conditions physiologiques, ils pourraient être actifs de façon tonique pendant les périodes de la sécrétion des glandes fundiques. Or l'hyperacidité provoque chez le mouton décérébré l'apparition des contractions du réticulo-rumen (Titchen, 1960) et l'augmentation de l'activité des centres gastriques et des neurones gastriques vagaux efférents (Harding et Leek, 1973 ; Leek et Harding, 1975). 
A la suite de cette revue, on constate que les différents types de récepteurs identifiés, ainsi que la nature de leur stimulus permettent d'expliquer l'origine des réflexes étudiés tant chez le mouton éveillé que chez l'animal anesthésié ou décérébré. L'analyse des récepteurs menée dans des conditions strictement physiologiques conduit-elle à des convergences avec celle réalisée sous anesthésie ? Les résultats actuels concernent les récepteurs du réticulo-rumen (Rousseau et Falempin, 1979 ; Falempin, 1981 ; Marie, Falempin et Rousseau, 1984). Un faible pourcentage de récepteurs (moins de $10 \%$ ) présentent une activité tonique qui augmente pendant les cycles primaires et secondaires. La distension du rumen par un gaz inerte provoque un accroissement de la fréquence de leur décharge et active en plus des récepteurs du même type, de seuil plus élevé, puisque silencieux dans les conditions physiologiques. Sensibles à la distension et à la contraction, ces récepteurs toniques sont vraisemblablement des récepteurs de tension à adaptation lente, en " série ", avec les éléments musculaires. Nous pouvons rattacher à cette classe les quelques récepteurs dont la décharge permanente cesse lors des événements moteurs du réticulo-rumen (Marie, Falempin et Rousseau, 1984). Bien qu'il ait été impossible de les localiser, on peut supposer qu'ils innervent des structures contractiles se relâchant pendant les cycles moteurs des préestomacs. La majorité des récepteurs gastriques étudiés présentent au contraire une activité phasique. Leur décharge en une ou deux bouffées de potentiels est associée avec la contraction biphasique du réseau ou avec les contractions primaires et secondaires du rumen. Elle n'est pas modifiée par la distension du rumen. Elle est parfois même diminuée pour certains récepteurs. La compression des parois du rumen, réalisée chez l'animal éveillé par l'intermédiaire d'une large fistule, stimule des récepteurs de ce type. Leur localisation et leur stimulus spécifique sont encore à préciser. Pour assimiler ces récepteurs à ceux épithéliaux du réticulo-rumen, il faut montrer qu'ils sont superficiels et également sensibles aux solutions acides. Comme leur décharge est phasique et contemporaine du mouvement des ingesta déterminé par les contractions, ils peuvent être soit strictement des mécanorécepteurs renseignant les centres sur l'évolution du transit - ce serait alors une nouvelle classe de récepteurs (dits de transit) - soit des récepteurs polymodaux (mécano et chémosensibles) identiques aux récepteurs épithéliaux, dont le seuil de réponse aux solutions acides est plus élevé que les concentrations normales d'AGV dans le rumen, ce qui expliquerait leur silence entre les contractions et l'allure phasique de leur décharge pendant celles-ci.

\section{Les centres gastriques bulbaires}

\section{Organisation des centres gastriques.}

La motricité du réticulo-rumen résulte de la mise en jeu de centres bulbaires gastriques, vers lesquels se projettent les informations sensitives d'origine périphérique. Ces centres sont localisés par des expériences de sections étagées du tronc cérébral (Dussardier et Albe-Fessard, 1954 ; Iggo, 1956 ; Titchen, 1958), de lésions électrolytiques (Beghelli et al., 1964) et de stimulations ponctuelles intrabulbaires (Dussardier, 1960 ; Howard, 1970). En comparant les effets de ces der- 
nières avec les différents types de réponse obtenus par la stimulation de la voie motrice ou de la voie sensitive vagale, Dussardier distinguait ainsi dans le bulbe des points moteurs directs, des points accélérateurs du rythme spontané et des points inhibiteurs de ce rythme. Plus tard, Harding et Leek (1971, 1982b, 1973) ont recueilli l'activité de neurones des centres gastriques en explorant systématiquement le bulbe à l'aide de microélectrodes. Ils ont ainsi mis en évidence l'existence de 3 types d'activités : des activités " afferent-like " modulées par les stimulations des différents récepteurs gastriques, des activités d'interneurones et des activités de neurones effecteurs. Les neurones « afferent-like " sont situés au voisinage des interneurones, dorso-latéralement par rapport à ces derniers. Les neurones effecteurs sont distingués des interneurones par la technique de collision antidromique et extériorisent une activité identique à celle recueillie sur les fibres vagales efférentes (cf. I). Ils correspondent aux corps cellulaires des neurones préganglionnaires vagaux et sont localisés dans le noyau dorsal du vague, là où Dussardier obtient, par stimulation, des réponses motrices directes. Les deux noyaux dorsaux du vague constituent donc l'étage effecteur du centre qui contrôle la motricité du réticulo-rumen. Les interneurones, tout comme les neurones préganglionnaires vagaux, extériorisent une activité périodique en relation avec les contractions gastriques. Ils sont de 3 types. Les décharges des interneurones de type $A$ ressemblent à celles des neurones préganglionnaires vagaux ; elles apparaissent de façon phasique plus ou moins précocément par rapport au début du cycle primaire ; elles sont modifiées par les influx sensitifs vagaux qui provoquent normalement des modifications de l'amplitude des contractions. Ces interneurones seraient situés fonctionnellement juste en amont des neurones préganglionnaires vagaux. Les interneurones de types $B$ et $C$ montrent une décharge soutenue qui, pour ceux du type $B$, augmente pendant la phase inactive entre 2 cycles primaires et atteint un maximum juste avant le début du cycle suivant ou qui, pour ceux du type $C$, cesse au début de la contraction réticulaire et pendant la durée du cycle. Leur activité, à la différence de celle des interneurones de type $A$, est peu influencée par les manœuvres expérimentales qui modifient de façon réflexe l'amplitude des contractions.

Selon la conception de Harding et Leek (1972b), les interneurones constituent l'étage intégrateur des centres gastriques, qui contrôle l'étage effecteur. Les interneurones de type $B$ déterminent la durée de la période silencieuse séparant deux cycles moteurs successifs, et donc leur fréquence. Ils constituent la base d'un " réseau fréquence " qui intègre les afférences périphériques et surtout centrales, de façon cumulative, jusqu'à ce qu'un niveau d'excitation soit atteint, qui permette l'expression du cycle suivant. Les interneurones de type $C$ du " réseau fréquence " inhibent les interneurones de type $A$ jusqu'à la fin de la période inactive, avant la séquence primaire suivante. A la levée de cette inhibition, les interneurones de type $A$ influencés de façon instantanée et directe par les afférences vagales, contrôlent l'amplitude et la forme des contractions par l'intermédiaire des neurones préganglionnaires vagaux. L'étage effecteur vagal est donc sous la dépendance d'un étage intégrateur composé de deux réseaux neuroniques. Le " réseau fréquence " (interneurones de types $B$ et $C$ ) en comptabilisant les informations qu'il reçoit des centres et de la périphérie, détermine le rythme des cycles 
primaires. II piloterait le " réseau forme et amplitude » (interneurones de type A), qui ajuste à tout moment la décharge de ses neurones en fonction des entrées vagales et permet, par l'intermédiaire des noyaux dorsaux du vague, l'expression de contractions dont l'intensité est adaptée aux modifications périphériques. Les activités cycliques des deux centres gastriques droit et gauche sont synchronisées grâce à des connexions commissurales entre les deux hémibulbes.

\section{Róle des afférences vagales.}

Le rythme des contractions du réticulo-rumen ne fait que traduire l'existence d'un rythme de même frequence des centres gastriques bulbaires. Ce rythme résulte-t-il de l'intégration au niveau central des afférences vagales provenant de l'estomac, celles-ci ayant un véritable rôle d'élaboration, ou bien les afférences vagales ne font-elles que " moduler " le rythme d'un oscillateur central préexistant, en jouant seulement un rôle de régulation ? Des éléments de réponse en faveur de la seconde hypothèse sont apportés par les expériences de déafférentation vagale (Falempin et Rousseau, 1979 ; Falempin, 1981). II est possible de détruire les corps cellulaires des neurones sensitifs vagaux au niveau du ganglion plexiforme, en laissant intacts les faisceaux de fibres efférentes. Comme la destruction bilatérale des ganglions entraîne la mort de l'animal, on associe à la section des deux branches thoraciques dorsale et ventrale d'un même vague, qui ne modifie par le rythme gastrique, la destruction du ganglion plexiforme controlatéral. Les centres bulbaires ne reçoivent plus d'afférences vagales, mais sont encore capables d'exprimer leur activité par un seul vague efférent. Trois résultats sont remarquables et constants.

- Nous observons tout d'abord la persistance du rythme gastrique, qui n'est donc pas élaboré par les afférences vagales, mais vraisemblablement par un oscillateur central. On peut supposer que celui-ci est constitué par les interneurones bulbaires de l'étage intégrateur, bien que leur activité cyclique cesse, lorsque sont interrompues sur la même préparation les afférences facilitatrices centrales et périphériques. Dans les conditions expérimentales d'Harding et Leek (1972b), cet arrêt pourrait être en effet la conséquence de la dépression de l'excitabilité des centres par l'anesthésie ; cette dépression mettrait au silence des neurones normalement autorythmiques qui n'ont plus leurs connexions afférentes.

- La fréquence des cycles primaires augmente après la déafférentation vagale et peut atteindre jusqu'à $150 \%$ de la fréquence initiale. Ce résultat est plutôt inattendu, puisque les afférences vagales gastriques sont surtout connues par leur effet dynamogène. En fait, on doit comprendre que l'activité rythmique des centres gastriques est en permanence déprimée par des afférences à effet inhibiteur, qui maintiennent la fréquence des cycles aux environs d'un par minute. A partir de ce niveau, une accélération du rythme gastrique peut alors résulter soit de la mise en jeu d'afférences dynamogènes, soit d'une diminution de la volée afférente inhibitrice. Nous avons vu dans le chapitre précédent la nature des stimulations responsables des effets moteurs ou inhibiteurs sur la motricité du réticulo-rumen. On peut postuler l'hypothèse d'un frein permanent des centres, exercé par la décharge tonique des récepteurs épithéliaux, les plus sensibles aux AGV. 
- La séquence ordonnée des contractions du réseau et du rumen, au cours d'un cycle primaire, est parfaitement respectée, ce qui est en faveur de sa programmation centrale. On peut supposer que, tout comme le centre de la déglutition (Jean, 1978), les centres gastriques sont polarisés et assurent dans un ordre déterminé, préétabli et indépendant de la volée afférente vagale, la mise en jeu des neurones qui innervent les structures du réticulo-rumen successivement impliquées dans le cycle primaire. Par contre, les cycles secondaires n'apparaissent plus et l'éructation est supprimée, ce qui montre l'importance des afférences vagales dans le déterminisme de la motricité permettant normalement l'élimination des gaz.

\section{Les niveaux de régulation}

Le tronc cérébral constitue le niveau d'intégration responsable de l'élaboration du rythme nerveux qui, par la voie efférente vagale, détermine celui des cycles primaires. Les afférences vagales sont essentielles pour régler le niveau de ce rythme, et l'amplitude des contractions, en fonction des stimulations périphériques d'origine gastrique. Des afférences trigéminales en provenance des récepteurs tactiles buccaux sont aussi capables de stimuler les centres gastriques, puisqu'une hypermotricité immédiate est observée dès le début des repas (Borgatti et Matscher, 1958). Bien que les centres gastriques reçoivent des influences provenant de centres situés au-dessus du tronc cérébral, peut-être de l'hypothalamus (Andersson, 1951), on peut conclure que le rythme et l'amplitude des contractions gastriques dépendent surtout de la mise en jeu de réflexes longs, pour la plupart vago-vagaux. Or des contractions dites intrinsèques, apparaissent en une à deux semaines après la bivagotomie (Ruckebusch, Tsiamitas et Bueno, 1972 ; Gregory, 1982) ; elles sont recueillies presque simultanément sur tout le réticulumrumen (Gregory, 1982), ce qui montre bien qu'une région " pace-maker » supportant l'hypothèse d'une organisation périphérique de la motricité chez le mouton intact, n'existe pas. Cette motricité intrinsèque dépend de l'activation des plexus intrapariétaux au sein desquels se réalisent des réflexes courts (Gregory, 1984a). Elle apparaît en effet chez le mouton bivagotomisé, lorsque le volume du rumen dépasse le seuil de 0,5-3 litres. Pour des distensions supraliminaires, la fréquence des contractions localisées augmente avec le niveau de la distension, et la réponse disparaît toujours sous atropine ou sous hexaméthonium. La motricité intrinsèque est abolie chez le mouton bivagotomisé par des concentrations élevées d'AGV, du même ordre que celles inhibant les contractions biphasiques du réseau et les contractions secondaires du rumen, et deux à trois fois plus fortes que celles inhibant les contractions primaires (Gregory, 1984b). Ces résultats montrent l'existence de mécanismes locaux de régulation dont le rôle dans les conditions physiologiques reste à préciser, par rapport à celui des mécanismes centraux. Des observations permettent cependant d'apprécier la nature des interrelations entre les deux niveaux de régulation. (i) Les récepteurs de tension du réticulo-rumen sont stimulés par les contractions intrinsèques (Iggo, 1955 ; Leek, 1969). Leur décharge de base est abolie, lorsque ces contractions intrinsèques 
sont absentes (Leek et Van Miert, 1971). Toute modification de la motricité intrinsèque induite par des réflexes locaux en réponse à une distension, entraîne des changements dans l'émission des récepteurs de tension, qui modulent alors de façon réflexe le rythme des contractions extrinsèques. Tout se passe comme si la motricité intrinsèque exerçait une influence indirecte sur la motricité extrinsèque. (ii) La déafférentation vagale fait apparaître dans un cycle plusieurs contractions de moindre amplitude que les contractions primaires du rumen, mais nettement plus fortes que les contractions intrinsèques observées chez le mouton intact (Falempin, 1981). Les afférences vagales auraient aussi pour rôle de limiter par voie réflexe l'importance de la motricité intrinsèque.

Ce problème de la dualité des niveaux de régulation se pose avec acuité, lorsqu'on étudie l'action de substances pharmacologiques, de neuromédiateurs ou d'hormones gastro-intestinales (revue dans Ruckebusch, 1983). En plus d'un effet direct sur les centres nerveux, les substances peuvent intervenir de plusieurs façons au niveau périphérique, (i) en agissant directement sur la sensibilité des terminaisons nerveuses réceptrices, (ii) indirectement en modulant la libération du neurotransmetteur par les neurones des plexus intrapariétaux, ce qui entraîne des modifications de la motricité et partant de la sensibilité des récepteurs de tension, (iii) en agissant directement sur la perméabilité membranaire des fibres musculaires, (iv) par plus d'un de ces mécanismes (Cottrell et lggo, 1984). Pour préciser les différents modes d'action d'une substance, l'enregistrement des efférences et afférences gastriques s'impose, en même temps que celui de l'activité électromyographique ou mécanique du viscère (Grovum et Leek, 1982). De même, l'utilisation de la méthode des sutures nerveuses croisées chez le mouton éveillé devrait être d'un intérêt certain dans de telles études concernant les modes d'action des peptides gastro-intestinaux.

Journées Ingestion, Digestion, Absorption de l'Association française de Nutrition, Versailles, 24-25 octobre 1984.

Remerciements. - Nous remercions vivement Mme C. Plancq pour la qualité de son travail, lors de la dactylographie des ébauches successives de ce rapport.

\section{Références}

ANDERSSON B., 1951. The effect and localization of electrical stimulation of certain parts of the brain stem in sheep and goats. Acta physiol. scand., 23, 8-23.

ASH R. W., KAY R. N. B., 1959. Stimulation and inhibition of reticulum contractions, rumination and parotid secretion from the forestomach of conscious sheep. J. Physiol. (London), 149, 43-57.

BEGHELLI V., BORGATTI G., MAVRULIS A., PARMEGGIANI P. L., 1964. Sulla organizzazione del centro reflesso del reticulo nell ovino. Arch. Sci. Biol., 48, 249-266.

BEGHELLI V., BORGATTI G., PARMEGGIANI P. L., 1963. On the role of the dorsal nucleus of the vagus in the reflex activity of the reticulum. Arch. ital. Biol., 101, 365-384.

BORGATTI G., MATSCHER R., 1958. Voies et signification du réflexe oral du réseau. Arch. ital. Biol., 96, 38-57. 
COTTRELL D. F., IGGO A., 1984. The responses of duodenal tension receptors in sheep to pentagastrin, cholecystokinin and some others drugs. J. Physiol. (London), 354, 477-495.

CRICHLOW E. C., LEEK B. F., 1980. The importance of $\mathrm{pH}$ in relation to the acid-excitation of epithelial receptors in the reticulo-rumen of sheep. J. Physiol. (London), 310, 60-61 P.

CRICHLOW E. C., CHAPLIN R. K., 1984. Forestomach epithelial receptor activation by grain overload rumen fluids. Can. J. anim. sci., 64 (Suppl.), 5-7.

DUSSARDIER M., 1958. La commande motrice de l'estomac étudiée chez le Mouton par la technique de la suture pneumogastrique-phrénique. J. Physiol. (Paris), 50, 265-268.

DUSSARDIER M., 1960. Recherches sur le controle bulbaire de la motricité gastrique chez les Ruminants. Th. Doct. ès Sci., Paris, I.N.R.A. Ed., 196 p.

DUSSARDIER M., ALBE-FESSARD D., 1954. Quelques propriétés du centre vagal contrôlant l'activité réflexe de l'estomac des Ruminants. J. Physiol. (Paris), 46, 354-357.

FALEMPIN M., 1981. Contribution à l'étude des afférences vagales digestives chez l'animal éveillé. Th. Doct. ès Sci., Lille, $196 \mathrm{p}$.

FALEMPIN M., MEI N., ROUSSEAU J. P., 1978. Vagal mechanoreceptors of the inferior thoracic oesophagus, the lower oesophageal sphincter and the stomach in the sheep. Pflügers Arch., 373, 25-30.

FALEMPIN M., ROUSSEAU J. P., 1979. Vagal digestive deafferentation in sheep. Ann. Rech. vét., 10, $186-188$.

FALEMPIN M., ROUSSEAU J. P., 1983. Reinnervation of skeletal muscles by vagal sensory fibres in the sheep, cat and rabbit. J. Physiol. (London), 335, 467-479.

GREGORY P. C., 1982. Forestomach motility in the chronically vagotomized sheep. J. Physiol. (London), 328, 431-447.

GREGORY P. C., 1984a. Control of intrinsic reticulo-rumen motility in the vagotomized sheep. J. Physiol. (London), 346, 379-393.

GREGORY P. C., 1984b. Inhibition of forestomach motility by short-chain volatile fatty acids in sheep. Can. J. anim. Sci., 64 (Suppl.), 11-12.

GROVUM W. L., LEEK B. F., 1982. Preliminary evidence of some central nervous, peripheral and reflex actions of pentagastrin on gastric motility. Irish J. med. Sci., 151, 86.

HARDING R., LEEK B. F., 1971. The location and activities of medullary neurones associated with ruminant forestomach motility. J. Physiol. (London), 219, 587-610.

HARDING R., LEEK B. F., 1972a. Rapidly adapting mechanoreceptors in the reticulo-rumen which also respond to chemicals. J. Physiol. (London), 223, 32-33 P.

HARDING R., LEEK B. F., 1972b. The effects of peripheral and central nervous influences on gastric centre neuronal activity in sheep. J. Physiol. (London), 225, 309-328.

HARDING R., LEEK B. F., 1973. Central projections of gastric afferent vagal inputs. J. Physiol. (London), 228, 73-90.

HOWARD B. R., 1968. Neuronal activity in the dorsal vagal nucleus of the sheep. J. Physiol. (London), 198, 111 P.

HOWARD B. R., 1970. The dorsal nucleus of the vagus as a center controlling gastric motility in sheep. J. Physiol. (London), 206, 167-180.

IGGO A., 1955. Tension receptors in the stomach and the urinary bladder. J. Physiol. (London), $128,593-607$.

IGGO A., 1956. Central nervous control of gastric movements in sheep and goats. J. Physiol. (London), 131, 248-256.

IGGO A., LEEK B. F., 1967. An electrophysiological study of single vagal efferent units associated with gastric movements in sheep. J. Physiol. (London), 191, 177-204.

JEAN A., 1978. Controle bulbaire de la déglutition et de la motricité aesophagienne. Th. Doct. ès Sci., Marseille, $278 \mathrm{p}$.

LEEK B. F., 1963. Single unit activity in cervical vagal efferent axons associated with reticuloruminal movements. J. Physiol. (London), 169, 5-6 P.

LEEK B. F., 1969. Reticulo-ruminal mechanoreceptors in sheep. J. Physiol. (London), 202, 585-609.

LLEK B. F., 1971. Abdominal visceral receptors, 113-160. In NEIL E., Handbook of sensory physiology, Vol. III/1. Enteroceptors, Springer Verlag, Berlin. 
LEEK B. F., 1972. The innervation of sheep forestomach papillae from which combined chemoreceptor and rapidly adapting mechanoreceptor response are obtainable. J. Physiol. (London), 227, 22-23 P.

LEEK B. F., HARDING R. H., 1975. Sensory nervous receptors in the ruminant stomach and the reflex control of reticulo-ruminal motility. In Mc DONALD I. W., WARNER A. C. I., Digestion and metabolism in the ruminant, Univ. New England Publ. Unit, Armidale, 60-76.

LEEK B. F., RYAN J. P., UPTON P. K., 1978. On the greater potency of butyric acid in inhibiting ruminant stomach (reticulo-ruminal) movements. J. Physiol. (London), 284, 158-159 P.

LEEK B. F., VAN MIERT A. S. J. P. A. M., 1971. An analysis of the gastric stasis induced by pyrogen : the effects of intrinsic and extrinsic movements of the ruminant forestomach. Rendic. R. Gastroenterol., 3, 163-167.

MARIE A., FALEMPIN M., ROUSSEAU J. P., 1984. Activity of esophageal and gastric receptors in conscious sheep. Can. J. anim. Sci., 64 (Suppl.), 1-2.

MORRISON A. R., HABEL R. E., 1964. A quantitative study of the distribution of vagal nerve endings in the myenteric plexus of the ruminant stomach. J. comp. Neurol., 122, 297-309.

NEWHOOK J. C., TITCHEN D. A., 1972. Effects of stimulation of efferent fibres of the vagus on the reticulo-omasal orifice of the sheep. J. Physiol. (London), 222, 407-418.

REID A. M., TITCHEN D. A., 1984. Effects of vasoactive intestinal polypeptide on the reticuloomasal orifice of the lamb. Can. J. anim. Sci., 64 (Suppl.), 91-92.

ROUSSEAU J.P., 1984. Electrophysiological study of vagal afferent and efferent units in conscious sheep. Quart. J. exper. Physiol., 69, 627-637.

ROUSSEAU J. P., FALEMPIN M., 1979. Activity of gut receptors in the conscious sheep. Ann. Rech. vét., 10, 189-191.

ROUSSEAU J. P., FALEMPIN M., 1984. Reinnervation of a striated muscle by vagal sensory axons. J. Auton. Nerv. Syst., 10, 217-223.

RUCKEBUSCH Y., 1983. Pharmacology of reticulo-ruminal motor function. J. vet. Pharmacol. Therap., 6, 245-272.

RUCKEBUSCH Y., KAY R. N. B., 1971. Etude critique de la motricité gastrique chez les Bovins. Ann. Rech. vét., 2, 99-136.

RUCKEBUSCH Y., TSIAMITAS C. H., BUENO L., 1972. The intrinsic electrical activity of the ruminant stomach. Life Sci., Oxford, 11, 55-64.

TITCHEN D. A., 1958. Reflex stimulation and inhibition of reticulum contractions in the ruminant stomach. J. Physiol. (London), 141, 1-21.

TITCHEN D. A., 1960. The production of rumen and reticulum contractions in decerebrate preparations of sheep and goats. J. Physiol. (London), 151, 139-153. 